\title{
Stability of Various Functional Equations in Non-Archimedean Intuitionistic Fuzzy Normed Spaces
}

\section{Syed Abdul Mohiuddine, Abdullah Alotaibi, and Mustafa Obaid}

Department of Mathematics, Faculty of Science, King Abdulaziz University, P.O. Box 80203, Jeddah 21589, Saudi Arabia

Correspondence should be addressed to Syed Abdul Mohiuddine, mohiuddine@gmail.com

Received 18 May 2012; Revised 25 October 2012; Accepted 9 November 2012

Academic Editor: Seenith Sivasundaram

Copyright (C) 2012 Syed Abdul Mohiuddine et al. This is an open access article distributed under the Creative Commons Attribution License, which permits unrestricted use, distribution, and reproduction in any medium, provided the original work is properly cited.

\begin{abstract}
We define and study the concept of non-Archimedean intuitionistic fuzzy normed space by using the idea of $t$-norm and $t$-conorm. Furthermore, by using the non-Archimedean intuitionistic fuzzy normed space, we investigate the stability of various functional equations. That is, we determine some stability results concerning the Cauchy, Jensen and its Pexiderized functional equations in the framework of non-Archimedean IFN spaces.
\end{abstract}

\section{Introduction}

The study of stability problem of functional equations originated from a question of Ulam [1] concerning the stability of group homomorphisms.

Let $(G, *)$ be a group and let $\left(G^{\prime}, \circ, d\right)$ be a metric group with the metric $d(\cdot, \cdot)$. Given $\epsilon>0$, does there exist a $\delta(\epsilon)>0$ such that if a mapping $h: G \rightarrow G^{\prime}$ satisfies the inequality $d(h(x * y), h(x) \circ h(y))<\delta$ for all $x, y \in G$, then there exists a homomorphism $H: G \rightarrow G^{\prime}$ with $d(h(x), H(x))<\epsilon$ for all $x \in G$ ?

If the answer is affirmative, we would say that the equation of homomorphism $H(x * y)=H(x) \circ H(y)$ is stable. The concept of stability for a functional equation arises when we replace the functional equation by an inequality which acts as a perturbation of the equation. Hyers [2] gave a first affirmative partial answer to the question of Ulam for Banach spaces. Hyers theorem was generalized by Aoki [3] for additive mappings and by Rassias [4] for linear mappings by considering an unbounded Cauchy difference. The paper of Rassias has significantly influenced the development of what we now call the Hyers-UlamRassias stability of functional equations. Since then several stability problems for various functional equations have been investigated in [5-20]. Quite recently, the stability problem 
for Pexiderized quadratic functional equation, Jensen functional equation, cubic functional equation, functional equations associated with inner product spaces, and additive functional equation was considered in [21-26], respectively, in the intuitionistic fuzzy normed spaces; while the idea of intuitionistic fuzzy normed space was introduced in [27] and further studied in [28-34] to deal with some summability problems. Quite recently, Alotaibi and Mohiuddine [35] established the stability of a cubic functional equation in random 2-normed spaces, while the notion of random 2-normed spaces was introduced by Goleț [36] and further studied in [37-39].

By modifying the definition of intuitionistic fuzzy normed space [27], in this paper, we introduce the notion of non-Archimedean intuitionistic fuzzy normed space and also establish Hyers-Ulam-Rassias-type stability results concerning the Cauchy, Pexiderized Cauchy, Jensen, and Pexiderized Jension functional equations in this new setup. This work indeed presents a relationship between four various disciplines: the theory of fuzzy spaces, the theory of non-Archimedean spaces, the theory of Hyers-Ulam-Rassias stability, and the theory of functional equations.

\section{Non-Archimedean Intuitionistic Fuzzy Normed Space}

In this section, we introduce the concept of non-Archimedean intuitionistic fuzzy normed space and further define the notions of convergence and Cauchy sequences in this new framework. We will assume throughout this paper that the symbols $\mathbb{N}, \mathbb{R}, \mathbb{C}$, and $\mathbb{Q}$ will denote the set of all natural, real, complex, and rational numbers, respectively.

A valuation is a map $|\cdot|$ from a field $\mathbb{K}$ into $[0, \infty)$ such that 0 is the unique element having the 0 valuation, $\left|k_{1} k_{2}\right|=\left|k_{1}\right|\left|k_{2}\right|$, and the triangle inequality holds, that is, $\left|k_{1}+k_{2}\right| \leq$ $\left|k_{1}\right|+\left|k_{2}\right|$, for all $k_{1}, k_{2} \in \mathbb{K}$. We say that a field $\mathbb{K}$ is valued if $\mathbb{K}$ carries a valuation. The usual absolute values of $\mathbb{R}$ and $\mathbb{C}$ are examples of valuations.

Let us consider a valuation which satisfies stronger condition than the triangle inequality. If the triangle inequality is replaced by $\left|k_{1}+k_{2}\right| \leq \max \left\{\left|k_{1}\right|,\left|k_{2}\right|\right\}$, for all $k_{1}, k_{2} \in \mathbb{K}$ then, a map $|\cdot|$ is called non-Archimedean or ultrametric valuation, and field is called a non-Archimedean field. Clearly $|1|=|-1|=1$ and $|n| \leq 1$, for all $n \in \mathbb{N}$. A trivial example of a non-Archimedean valuation is the map $|\cdot|$ taking everything but 0 into 1 and $|0|=0$.

Let $X$ be a vector space over a field $\mathbb{K}$ with a non-Archimedean valuation $|\cdot|$. A nonArchimedean normed space is a pair $(X,\|\cdot\|)$, where $\|\cdot\|: X \rightarrow[0, \infty)$ is such that

(i) $\|x\|=0$ if and only if $x=0$,

(ii) $\|\alpha x\|=|\alpha|\|x\|$ for $\alpha \in \mathbb{K}$, and

(iii) the strong triangle inequality, $\|x+y\| \leq \max \{\|x\|,\|y\|\}$, for $x, y \in X$.

In [40], Hensel discovered the $p$-adic numbers as a number theoretical analogue of power series in complex analysis. The most interesting example of non-Archimedean spaces is $p$-adic numbers.

Example 2.1. Let $p$ be a prime number. For any nonzero rational number $a=p^{r} m / n$ such that $m$ and $n$ are coprime to the prime number $p$, define the $p$-adic absolute value $|a|_{p}=p^{-r}$. Then $|\cdot|$ is a non-Archimedean norm on $\mathbb{Q}$. The completion of $\mathbb{Q}$ with respect to $|\cdot|$ is denoted by $\mathbb{Q}_{p}$ and is called the $p$-adic number field. 
A binary operation $*:[0,1] \times[0,1] \rightarrow[0,1]$ is said to be a continuous $t$-norm if it satisfies the following conditions.

(a) $*$ is associative and commutative, (b) $*$ is continuous, (c) $a * 1=a$ for all $a \in[0,1]$, and (d) $a * b \leq c * d$ whenever $a \leq c$ and $b \leq d$ for each $a, b, c, d \in[0,1]$.

A binary operation $\diamond:[0,1] \times[0,1] \rightarrow[0,1]$ is said to be a continuous t-conorm if it satisfies the following conditions.

$\left(\mathrm{a}^{\prime}\right) \diamond$ is associative and commutative, $\left(\mathrm{b}^{\prime}\right) \diamond$ is continuous, $\left(\mathrm{c}^{\prime}\right) a \diamond 0=a$ for all $a \in[0,1]$, and $\left(\mathrm{d}^{\prime}\right) a \diamond b \leq c \diamond d$ whenever $a \leq c$ and $b \leq d$ for each $a, b, c, d \in[0,1]$.

Definition 2.2. The five-tuple $(X, \varepsilon, \mathcal{F}, *, \diamond)$ is said to be an non-Archimedean intuitionistic fuzzy normed space (for short, non-Archimedean IFN space) if $X$ is a vector space over a nonArchimedean field $\mathbb{K}, *$ is a continuous t-norm, $\diamond$ is a continuous $t$-conorm, and $\mathcal{E}, \mathcal{F}$ are functions from $X \times \mathbb{R}$ to $[0,1]$ satisfying the following conditions. For every $x, y \in X$ and $s, t \in \mathbb{K}$ (i) $\mathcal{\varepsilon}(x, t)+\mathcal{F}(x, t) \leq 1$, (ii) $\mathcal{\varepsilon}(x, t)>0$, (iii) $\mathcal{\varepsilon}(x, t)=1$ if and only if $x=0$, (iv) $\mathcal{\varepsilon}(\alpha x, t)=\mathcal{E}(x, t /|\alpha|)$ for each $\alpha \neq 0$, (v) $\mathcal{\varepsilon}(x, t) * \mathcal{\varepsilon}(y, s) \leq \mathcal{\varepsilon}(x+y$, $\max \{t, s\})$, (vi) $\mathcal{E}(x, \cdot):(0, \infty) \rightarrow[0,1]$ is continuous, (vii) $\lim _{t \rightarrow \infty} \mathcal{E}(x, t)=1$ and $\lim _{t \rightarrow 0} \mathcal{E}(x, t)=0$, (viii) $\mathcal{F}(x, t)<1$, (ix) $\mathcal{F}(x, t)=0$ if and only if $x=0,(x) \mathcal{F}(\alpha x, t)=\mathcal{F}(x, t /|\alpha|)$ for each $\alpha \neq 0$, (xi) $\mathcal{F}(x, t) \diamond \mathcal{F}(y, s) \geq \mathcal{F}(x+y, \max \{t, s\}),(x i i) \mathcal{F}(x, \cdot):(0, \infty) \rightarrow[0,1]$ is continuous, and (xiii) $\lim _{t \rightarrow \infty} \mathcal{F}(x, t)=0$ and $\lim _{t \rightarrow 0} \mathcal{F}(x, t)=1$.

In this case $(\mathcal{E}, \mathcal{F})$ is called a non-Archimedean intuitionistic fuzzy norm.

Example 2.3. Let $(X,\|\cdot\|)$ be a non-Archimedean normed space, $a * b=a b$ and $a \diamond b=\min \{a+$ $b, 1\}$ for all $a, b \in[0,1]$. For all $x \in X$, every $t>0$ and $k=1,2$, consider the following:

$$
\varepsilon_{k}(x, t)=\left\{\begin{array}{ll}
\frac{t}{t+k\|x\|} & \text { if } t>0, \\
0 & \text { if } t \leq 0 ;
\end{array} \quad \mathbb{F}_{k}(x, t)= \begin{cases}\frac{k\|x\|}{t+k\|x\|} & \text { if } t>0 \\
1 & \text { if } t \leq 0 .\end{cases}\right.
$$

Then $\left(X, \varepsilon_{k}, \mathcal{F}_{k}, *, \diamond\right)$ is a non-Archimedean intuitionistic fuzzy normed space.

Definition 2.4. Let $(X, \mathcal{\varepsilon}, \mathcal{F}, *, \diamond)$ be a non-Archimedean intuitionistic fuzzy normed space. Then, a sequence $s=\left(s_{n}\right)$ is said to be

(i) convergent in $(X, \mathcal{\varepsilon}, \mathcal{F}, *, \diamond)$ or simply $(\mathcal{E}, \mathcal{F})$-convergent to $\xi \in X$ if for every $\epsilon>0$ and $t>0$, there exists $n_{0} \in \mathbb{N}$ such that $\mathcal{E}\left(s_{n}-\xi, t\right)>1-\epsilon$ and $\mathcal{F}\left(s_{n}-\xi, t\right)<\epsilon$ for all $n \geq n_{0}$. In this case we write $(\boldsymbol{\xi}, \mathcal{F})-\lim _{n} s_{n}=\xi$ and $\xi$ is called the $(\boldsymbol{\xi}, \mathcal{F})$-limit of $s=\left(s_{n}\right)$.

(ii) Cauchy in $(X, \mathcal{\varepsilon}, \mathcal{F}, *, \diamond)$ or simply $(\mathcal{E}, \mathcal{F})$-Cauchy if for every $\epsilon>0$ and $t>0$, there exists $n_{0} \in \mathbb{N}$ such that $\mathcal{E}\left(s_{n}-s_{m}, t\right)>1-\epsilon$ and $\mathcal{F}\left(s_{n}-s_{m}, t\right)<\epsilon$ for all $n, m \geq n_{0}$. A non-Archimedean IFN-space $(X, \varepsilon, \mathcal{F}, *, \diamond)$ is said to be complete if every $(\mathcal{E}, \mathcal{F})$-Cauchy is $(\mathcal{E}, \mathcal{F})$-convergent. In this case $(X, \mathcal{\varepsilon}, \mathcal{F}, *, \diamond)$ is called nonArchimedean intuitionistic fuzzy Banach space.

\section{Stability of Cauchy Functional Equation}

In this section, we determine stability result concerning the Cauchy functional equation $f(x+$ $y)=f(x)+f(y)$ in non-Archimedean intuitionistic fuzzy normed space. 
Theorem 3.1. Let $X$ be a linear space over a non-Archimedean field $\mathbb{K}$ and let $\left(Z, \mathcal{E}^{\prime}, \mathcal{F}^{\prime}\right)$ be a nonArchimedean IFN space. Suppose that $\varphi: X \times X \rightarrow Z$ is a function such that for some $\alpha>0$ and some positive integer $k$ with $|k|<\alpha$

$$
\begin{aligned}
& \mathcal{E}^{\prime}\left(\varphi\left(k^{-1} x, k^{-1} y\right), t\right) \geq \mathcal{E}^{\prime}(\varphi(x, y), \alpha t), \\
& \mathcal{F}^{\prime}\left(\varphi\left(k^{-1} x, k^{-1} y\right), t\right) \leq \mathcal{F}^{\prime}(\varphi(x, y), \alpha t),
\end{aligned}
$$

for all $x, y \in X$ and $t>0$. Let $(Y, \varepsilon, \mathcal{F})$ be a non-Archimedean intuitionistic fuzzy Banach space over $\mathbb{K}$ and let $f: X \rightarrow Y$ be a $\varphi$-approximately Cauchy mapping in the sense that

$$
\begin{aligned}
& \mathcal{E}(f(x+y)-f(x)-f(y), t) \geq \mathcal{E}^{\prime}(\varphi(x, y), t), \\
& \mathcal{F}(f(x+y)-f(x)-f(y), t) \leq \mathcal{F}^{\prime}(\varphi(x, y), t),
\end{aligned}
$$

for all $x, y \in X$ and $t>0$. Then there exists a unique additive mapping $C: X \rightarrow Y$ such that

$$
\mathcal{E}(f(x)-C(x), t) \geq \mathcal{M}(x, \alpha t), \quad F(f(x)-C(x), t) \leq \mathcal{N}(x, \alpha t),
$$

for all $x \in X$ and $t>0$, where

$$
\begin{aligned}
& \mathcal{M}(x, t)=\mathcal{E}^{\prime}(\varphi(x, x), t) * \mathcal{E}^{\prime}(\varphi(x, 2 x), t) * \cdots * \mathcal{E}^{\prime}(\varphi(x,(k-1) x), t), \\
& \mathcal{N}(x, t)=\mathcal{F}^{\prime}(\varphi(x, x), t) \diamond \mathcal{F}^{\prime}(\varphi(x, 2 x), t) \diamond \cdots \diamond \mathcal{F}^{\prime}(\varphi(x,(k-1) x), t) .
\end{aligned}
$$

Proof. By induction on $j$ we will show that for each $x \in X, t>0$ and $j \geq 2$

$$
\begin{aligned}
& \mathcal{E}(f(j x)-j f(x), t) \geq \mathcal{M}_{j}(x, t)=\mathcal{E}^{\prime}(\varphi(x, x), t) * \cdots * \mathcal{E}^{\prime}(\varphi(x,(j-1) x), t), \\
& \mathcal{F}(f(j x)-j f(x), t) \leq \mathcal{N}_{j}(x, t)=\mathcal{F}^{\prime}(\varphi(x, x), t) \diamond \cdots \diamond \mathcal{F}^{\prime}(\varphi(x,(j-1) x), t) .
\end{aligned}
$$

Putting $x=y$ in (3.2), we obtain

$$
\mathcal{E}(f(2 x)-2 f(x), t) \geq \mathcal{E}^{\prime}(\varphi(x, x), t), \quad \mathcal{F}(f(2 x)-2 f(x), t) \leq \mathcal{F}^{\prime}(\varphi(x, x), t),
$$

for all $x \in X$ and $t>0$. This proves (3.5) for $j=2$. Let (3.5) hold for some $j>2$. Replacing $y$ by $j x$ in (3.2), we get

$$
\begin{aligned}
& \mathcal{\varepsilon}(f((j+1) x)-f(x)-f(j x), t) \geq \mathcal{E}^{\prime}(\varphi(x, j x), t), \\
& \mathcal{F}(f((j+1) x)-f(x)-f(j x), t) \leq \mathcal{F}^{\prime}(\varphi(x, j x), t),
\end{aligned}
$$


for each $x \in X$ and $t>0$. Thus

$$
\begin{aligned}
& \mathcal{E}(f((j+1) x)-(j+1) f(x), t) \\
&=\mathcal{E}(f((j+1) x)-f(x)-f(j x)+f(j x)-j f(x), t) \\
& \geq \mathcal{E}(f((j+1) x)-f(x)-f(j x), t) * \mathcal{E}(f(j x)-j f(x), t) \\
& \geq \mathcal{E}^{\prime}(\varphi(x, j x), t) * \mathcal{M}_{j}(x, t)=\mathcal{M}_{j+1}(x, t), \\
& \mathcal{F}(f((j+1) x)-(j+1) f(x), t) \\
& \quad=\mathscr{F}(f((j+1) x)-f(x)-f(j x)+f(j x)-j f(x), t) \\
& \quad \leq \mathcal{F}(f((j+1) x)-f(x)-f(j x), t) \diamond \mathcal{F}(f(j x)-j f(x), t) \\
& \quad \leq \mathcal{F}^{\prime}(\varphi(x, j x), t) \diamond \mathcal{N}_{j}(x, t)=\mathcal{N}_{j+1}(x, t),
\end{aligned}
$$

for each $x \in X$ and $t>0$. Hence (3.5) holds for all $j \geq 2$. In particular

$$
\mathcal{E}(f(k x)-k f(x), t) \geq \mathcal{M}(x, t), \quad \mp(f(k x)-k f(x), t) \leq \mathcal{N}(x, t) .
$$

Replacing $x$ by $k^{-n-1} x$ in (3.9) and using (3.1), we get

$$
\begin{aligned}
& \varepsilon\left(f\left(k^{-n} x\right)-k f\left(k^{-(n+1)} x\right), t\right) \geq \mathcal{M}\left(x, \alpha^{n+1} t\right), \\
& \mathcal{F}\left(f\left(k^{-n} x\right)-k f\left(k^{-(n+1)} x\right), t\right) \leq \mathcal{N}\left(x, \alpha^{n+1} t\right),
\end{aligned}
$$

for all $x \in X, t>0$ and $n=0,1,2, \ldots$. Therefore

$$
\begin{aligned}
& \mathcal{E}\left(k^{n} f\left(k^{-n} x\right)-k^{n+1} f\left(k^{-(n+1)} x\right), t\right) \geq \mathcal{M}\left(x, \frac{\alpha^{n+1} t}{|k|^{n}}\right), \\
& \mathcal{F}\left(k^{n} f\left(k^{-n} x\right)-k^{n+1} f\left(k^{-(n+1)} x\right), t\right) \leq \mathcal{N}\left(x, \frac{\alpha^{n+1} t}{|k|^{n}}\right),
\end{aligned}
$$

for all $x \in X, t>0$ and $n=0,1,2, \ldots$ Since

$$
\lim _{m \rightarrow \infty} \mathcal{M}\left(x, \frac{\alpha^{m+1} t}{|k|^{m}}\right)=1, \quad \lim _{m \rightarrow \infty} \mathcal{N}\left(x, \frac{\alpha^{m+1} t}{|k|^{m}}\right)=0,
$$

so (3.11) shows that $\left(k^{n} f\left(k^{-n} x\right)\right)$ is a Cauchy sequence in non-Archimedean intuitionistic fuzzy Banach space $(Y, \mathcal{\varepsilon}, \mathcal{F})$. Therefore, we can define a mapping $C: X \rightarrow Y$ by $C x=$ $(\varepsilon, \mathcal{F})-\lim _{n \rightarrow \infty} k^{n} f\left(k^{-n} x\right)$. Hence

$$
\lim _{n \rightarrow \infty} \mathcal{E}\left(k^{n} f\left(k^{-n} x\right)-C(x), t\right)=1, \quad \lim _{n \rightarrow \infty} \mathcal{F}\left(k^{n} f\left(k^{-n} x\right)-C(x), t\right)=0 .
$$


For each $n \geq 1, x \in X$ and $t>0$

$$
\begin{aligned}
\mathcal{E}\left(f(x)-k^{n} f\left(k^{-n} x\right), t\right) & =\mathcal{E}\left(\sum_{i=0}^{n-1} k^{i} f\left(k^{-i} x\right)-k^{i+1} f\left(k^{-(i+1)} x\right), t\right) \\
& \geq \prod_{i=0}^{n-1} \mathcal{\varepsilon}\left(k^{i} f\left(k^{-i} x\right)-k^{i+1} f\left(k^{-(i+1)} x\right), t\right) \\
& =\mathcal{M}(x, \alpha t), \\
\mathcal{F}\left(f(x)-k^{n} f\left(k^{-n} x\right), t\right) & \leq \coprod_{i=0}^{n-1} \mp\left(k^{i} f\left(k^{-i} x\right)-k^{i+1} f\left(k^{-(i+1)} x\right), t\right) \\
& =\mathcal{N}(x, \alpha t),
\end{aligned}
$$

where $\prod_{j=1}^{n} a_{j}=a_{1} * a_{2} * \cdots * a_{n}$ and $\coprod_{j=1}^{n} a_{j}=a_{1} \diamond a_{2} \diamond \cdots \diamond a_{n}$. It follows from (3.13) and (3.14) that

$$
\begin{aligned}
& \mathcal{E}(f(x)-C(x), t) \geq \mathcal{E}\left(f(x)-k^{n} f\left(k^{-n} x\right), t\right) * \mathcal{E}\left(k^{n} f\left(k^{-n} x\right)-C(x), t\right) \geq \mathcal{M}(x, \alpha t), \\
& \mathcal{F}(f(x)-C(x), t) \leq \mathcal{F}\left(f(x)-k^{n} f\left(k^{-n} x\right), t\right) \diamond \mathcal{F}\left(k^{n} f\left(k^{-n} x\right)-C(x), t\right) \leq \mathcal{N}(x, \alpha t),
\end{aligned}
$$

for each $x \in X, t>0$ and for sufficiently large $n$; that is, (3.3) holds. Also, from (3.1), (3.2), and (3.13), we have

$$
\begin{aligned}
& \mathcal{E}(C(x+y)-C(x)-C(y), t) \\
& \geq \mathcal{E}\left(C(x+y)-k^{n} f\left(k^{-n}(x+y)\right), t\right) * \mathcal{E}\left(k^{n} f\left(k^{-n} x\right)-C(x), t\right) \\
& * \mathcal{E}\left(k^{n} f\left(k^{-n} y\right)-C(y), t\right) * \mathcal{E}\left(k^{n} f\left(k^{-n}(x+y)\right)-k^{n} f\left(k^{-n} x\right)-k^{n} f\left(k^{-n} y\right), t\right) \\
& \geq \mathcal{E}^{\prime}\left(\varphi\left(k^{-n} x, k^{-n} y\right), \frac{t}{|k|^{n}}\right) \geq \mathcal{E}^{\prime}\left(\varphi(x, y), \frac{\alpha^{n} t}{|k|^{n}}\right), \\
& \mathcal{F}(C(x+y)-C(x)-C(y), t) \\
& \leq \mathcal{F}\left(C(x+y)-k^{n} f\left(k^{-n}(x+y)\right), t\right) \diamond \mathcal{F}\left(k^{n} f\left(k^{-n} x\right)-C(x), t\right) \\
& \diamond \mathcal{F}\left(k^{n} f\left(k^{-n} y\right)-C(y), t\right) \diamond \mathcal{F}\left(k^{n} f\left(k^{-n}(x+y)\right)-k^{n} f\left(k^{-n} x\right)-k^{n} f\left(k^{-n} y\right), t\right) \\
& \leq \mathcal{F}^{\prime}\left(\varphi\left(k^{-n} x, k^{-n} y\right), \frac{t}{|k|^{n}}\right) \geq \mathcal{F}^{\prime}\left(\varphi(x, y), \frac{\alpha^{n} t}{|k|^{n}}\right),
\end{aligned}
$$

for all $x, y \in X, t>0$ and for large $n$. Since

$$
\lim _{n \rightarrow \infty} \mathcal{E}^{\prime}\left(\varphi(x, y), \frac{\alpha^{n} t}{|k|^{n}}\right)=1, \quad \lim _{n \rightarrow \infty} \mathcal{F}^{\prime}\left(\varphi(x, y), \frac{\alpha^{n} t}{|k|^{n}}\right)=0,
$$


which shows that $C$ is additive. Now if $C^{\prime}: X \rightarrow Y$ is another additive mapping such that

$$
\mathcal{E}\left(C^{\prime}(x)-f(x), t\right) \geq \mathcal{M}(x, t), \quad \mathcal{F}\left(C^{\prime}(x)-f(x), t\right) \geq \mathcal{N}(x, t),
$$

for all $x \in X$ and $t>0$. Then, for all $x \in X, t>0$ and $n \in \mathbb{N}$, we have

$$
\begin{aligned}
\mathcal{E}\left(C(x)-C^{\prime}(x), t\right) & \geq \mathcal{E}\left(C(x)-k^{n} f\left(k^{-n} x\right), t\right) * \mathcal{E}\left(k^{n} f\left(k^{-n} x\right)-C^{\prime}(x), t\right) \\
& \geq \mathcal{E}\left(C\left(k^{-n} x\right)-f\left(k^{-n} x\right), \frac{t}{|k|^{n}}\right) * \mathcal{\varepsilon}\left(f\left(k^{-n} x\right)-C^{\prime}\left(k^{-n} x\right), \frac{t}{|k|^{n}}\right) \\
& \geq \mathcal{M}\left(k^{-n} x, \frac{\alpha t}{|k|^{n}}\right) \geq \mathcal{M}\left(x, \frac{\alpha^{n+1} t}{|k|^{n}}\right), \\
\mathcal{F}\left(C(x)-C^{\prime}(x), t\right) & \leq \mathcal{F}\left(C(x)-k^{n} f\left(k^{-n} x\right), t\right) \diamond \mathcal{F}\left(k^{n} f\left(k^{-n} x\right)-C^{\prime}(x), t\right) \\
& \leq \mathcal{F}\left(C\left(k^{-n} x\right)-f\left(k^{-n} x\right), \frac{t}{|k|^{n}}\right) \diamond \mathcal{F}\left(f\left(k^{-n} x\right)-C^{\prime}\left(k^{-n} x\right), \frac{t}{|k|^{n}}\right) \\
& \geq \mathcal{N}\left(k^{-n} x, \frac{\alpha t}{|k|^{n}}\right) \leq \mathcal{N}\left(x, \frac{\alpha^{n+1} t}{|k|^{n}}\right) .
\end{aligned}
$$

Therefore

$$
\lim _{n \rightarrow \infty} \mathcal{M}\left(x, \frac{\alpha^{n+1} t}{|k|^{n}}\right)=1, \quad \lim _{n \rightarrow \infty} \mathcal{N}\left(x, \frac{\alpha^{n+1} t}{|k|^{n}}\right)=0
$$

Hence $C(x)=C^{\prime}(x)$ for all $x \in X$.

Corollary 3.2. Let $X$ be a linear space over non-Archimedean field $\mathbb{K}$ and let $(Y,\|\cdot\|)$ be a non-Archimedean normed space. Suppose that a function $\varphi: X \times X \rightarrow \mathbb{R}^{+}$satisfies

$$
\varphi\left(k^{-1} x, k^{-1} y\right) \leq \alpha^{-1} \varphi(x, y)
$$

for all $x, y \in X$, where $\alpha>0$ and $k$ is an integer with $|k|<\alpha$. If a map $f: X \rightarrow Y$ satisfies

$$
\|f(x+y)-f(x)-f(y)\| \leq \varphi(x, y)
$$

for all $x, y \in X$, then there exists a unique additive mapping $C: X \rightarrow Y$ satisfies

$$
\|f(x)-C(x)\| \leq \frac{1}{\alpha} \max \{\varphi(x, x) * \varphi(x, 2 x) * \cdots * \varphi(x,(k-1) x)\} .
$$


Proof. Consider the non-Archimedean intuitionistic fuzzy norm

$$
\varepsilon(y, t)=\left\{\begin{array}{ll}
\frac{t}{t+\|y\|} & \text { if } t>0, \\
0 & \text { if } t \leq 0 ;
\end{array} \quad \mp(x, t)= \begin{cases}\frac{\|y\|}{t+\|y\|} & \text { if } t>0 \\
1 & \text { if } t \leq 0\end{cases}\right.
$$

on $Y$. Let $Z=\mathbb{R}$ and let the function $\mathcal{E}^{\prime}, \boldsymbol{F}^{\prime}: \mathbb{R} \times \mathbb{R} \rightarrow[0,1]$ be defined by

$$
\mathcal{E}^{\prime}(z, t)=\left\{\begin{array}{ll}
\frac{t}{t+|z|} & \text { if } t>0, \\
0 & \text { if } t \leq 0 ;
\end{array} \quad \mathcal{F}^{\prime}(z, t)= \begin{cases}\frac{|z|}{t+|z|} & \text { if } t>0, \\
1 & \text { if } t \leq 0 .\end{cases}\right.
$$

Then $\left(\mathcal{E}^{\prime}, \mathcal{F}^{\prime}\right)$ is a non-Archimedean intuitionistic fuzzy norm on $\mathbb{R}$. The result follows from the fact that (3.21), (3.22), and (3.23) are equivalent to (3.1), (3.2), and (3.3), respectively.

Example 3.3. Let $X$ be a linear space over non-Archimedean field $\mathbb{K}$ and let $(Y,\|\cdot\|)$ be a nonArchimedean normed space. Suppose that a function $f: X \rightarrow Y$ satisfies

$$
\|f(x+y)-f(x)-f(y)\| \leq\|x\|^{p}+\|y\|^{p},
$$

for all $x, y \in X$ and $p \in[0,1)$. Suppose that there exists an integer $k$ such that $|k|<1$. Since $p<1$, by applying Corollary 3.2 for $\varphi(x, y)=\|x\|^{p}+\|y\|^{p}$, we observe that (3.21) holds for $\alpha=|k|^{p}$. Inequality (3.23) assures the existence of a unique additive mapping $C: X \rightarrow Y$ such that

$$
\|f(x)-C(x)\| \leq \frac{1+(k-1)^{p}}{|k|^{p}}\|x\|^{p},
$$

for all $x \in X$.

\section{Stability of Pexiderized Cauchy Functional Equation}

The functional equation $f(x+y)=g(x)+h(y)$ is said to be Pexiderized Cauchy, where $f$, $g$, and $h$ are mappings between linear spaces. In the case $f=g=h$, it is called Cauchy functional equation.

Theorem 4.1. Let $X$ be a linear space over a non-Archimedean field $\mathbb{K}$ and let $(Y, \mathcal{\varepsilon}, \mathcal{F})$ be a nonArchimedean intuitionistic fuzzy Banach space. Suppose that $f, g$, and hare mappings from $X$ to $Y$ with $f(0)=g(0)=h(0)=0$. Suppose that $\varphi$ is a function from $X \times X$ to a non-Archimedean IFN space $\left(Z, \mathcal{E}^{\prime}, \mathcal{F}^{\prime}\right)$ such that

$$
\begin{aligned}
& \mathcal{E}(f(x+y)-g(x)-h(y), t) \geq \mathcal{E}^{\prime}(\varphi(x, y), t), \\
& \mathcal{F}(f(x+y)-g(x)-h(y), t) \leq \mathcal{F}^{\prime}(\varphi(x, y), t),
\end{aligned}
$$

for all $x, y \in X$ and $t>0$. If

$$
\mathcal{E}^{\prime}\left(\varphi\left(k^{-1} x, k^{-1} y\right), t\right) \geq \mathcal{E}^{\prime}(\varphi(x, y), \alpha t), \quad \mathcal{F}^{\prime}\left(\varphi\left(k^{-1} x, k^{-1} y\right), t\right) \leq \mathcal{F}^{\prime}(\varphi(x, y), \alpha t),
$$


for some positive real number $\alpha>0$ and some positive integer $k$ with $|k|<\alpha$, then there exists a unique additive mapping $C: X \rightarrow Y$ such that

$$
\begin{array}{cc}
\mathcal{E}(f(x)-C(x), t) \geq \mathcal{M}(x, \alpha t), & \mathcal{F}(f(x)-C(x), t) \leq \mathcal{N}(x, \alpha t), \\
\mathcal{E}(g(x)-C(x), t) \geq \mathcal{M}(x, \min \{1, \alpha\} t), & \mathcal{F}(g(x)-C(x), t) \leq \mathcal{N}(x, \min \{1, \alpha\} t), \\
\mathcal{E}(h(x)-C(x), t) \geq \mathcal{M}(x, \min \{1, \alpha\} t), & \mathcal{F}(h(x)-C(x), t) \leq \mathcal{N}(x, \min \{1, \alpha\} t),
\end{array}
$$

for all $x \in X$ and $t>0$, where

$$
\begin{aligned}
\mathcal{M}(x, t)= & \mathcal{E}^{\prime}(\varphi(x, x), t) * \cdots * \mathcal{E}^{\prime}(\varphi(x,(k-1) x), t) * \mathcal{E}^{\prime}(\varphi(0, x), t) * \cdots * \mathcal{E}^{\prime}(\varphi(0,(k-1) x), t) \\
& * \mathcal{E}^{\prime}(\varphi(x, 0), t) * \cdots * \mathcal{E}^{\prime}(\varphi((k-1) x, 0), t), \\
\mathcal{N}(x, t)= & \mathcal{F}^{\prime}(\varphi(x, x), t) \diamond \cdots \diamond \mathcal{F}^{\prime}(\varphi(x,(k-1) x), t) \diamond \mathcal{F}^{\prime}(\varphi(0, x), t) \diamond \cdots \diamond \mathcal{F}^{\prime}(\varphi(0,(k-1) x), t) \\
& \diamond \mathcal{F}^{\prime}(\varphi(x, 0), t) \diamond \cdots \diamond \mathcal{F}^{\prime}(\varphi((k-1) x, 0), t) .
\end{aligned}
$$

Proof. Put $y=0$ in (4.1). Then, for all $x \in X$ and $t>0$

$$
\mathcal{E}(f(x)-g(x), t) \geq \mathcal{E}^{\prime}(\varphi(x, 0), t), \quad F(f(x)-g(x), t) \leq \mathcal{F}^{\prime}(\varphi(x, 0), t) .
$$

For $x=0,(4.1)$ becomes

$$
\mathcal{E}(f(y)-h(y), t) \geq \mathcal{E}^{\prime}(\varphi(0, y), t), \quad \mathcal{F}(f(y)-h(y), t) \leq \mathcal{F}^{\prime}(\varphi(0, y), t),
$$

for all $y \in X$ and $t>0$. Combining (4.1), (4.7), and (4.8), we obtain

$$
\begin{aligned}
& \mathcal{E}(f(x+y)-f(x)-f(y), t) \geq \mathcal{E}^{\prime}(\varphi(x, y), t) * \mathcal{E}^{\prime}(\varphi(x, 0), t) * \mathcal{E}^{\prime}(\varphi(0, y), t), \\
& \mathcal{F}(f(x+y)-f(x)-f(y), t) \leq \mathcal{F}^{\prime}(\varphi(x, y), t) \diamond \mathcal{F}^{\prime}(\varphi(x, 0), t) \diamond \mathcal{F}^{\prime}(\varphi(0, y), t),
\end{aligned}
$$

for each $x, y \in X$ and $t>0$. Replacing $\mathcal{E}^{\prime}(\varphi(x, y), t)$ and $\mathcal{F}^{\prime}(\varphi(x, y), t)$ by $\mathcal{E}^{\prime}(\varphi(x, y), t) *$ $\mathcal{E}^{\prime}(\varphi(x, 0), t) * \mathcal{E}^{\prime}(\varphi(0, y), t)$ and $\mathcal{F}^{\prime}(\varphi(x, y), t) \Delta \mathcal{F}^{\prime}(\varphi(x, 0), t) \Delta \mathcal{F}^{\prime}(\varphi(0, y), t)$, respectively, in Theorem 3.1, we can find that there exists a unique additive mapping $C: X \rightarrow Y$ that satisfies (4.3). From (4.3) and (4.7), we see that

$$
\begin{aligned}
& \mathcal{E}(g(x)-T(x), t) \geq \mathcal{E}(g(x)-f(x), t) * \mathcal{E}(f(x)-T(x), t) \geq \mathcal{M}(x, t), \\
& \mathcal{F}(g(x)-T(x), t) \leq \mathcal{F}(g(x)-f(x), t) \diamond \mathcal{F}(f(x)-T(x), t) \leq \mathcal{N}(x, t),
\end{aligned}
$$

for all $x, y \in X$ and $t>0$, which proves (4.4). Similarly, we can prove (4.5).

Corollary 4.2. Let $X$ be a linear space over a non-Archimedean field $\mathbb{K}$ and let $\left(Z, \mathcal{\varepsilon}^{\prime}, \mathcal{F}^{\prime}\right)$ be a nonArchimedean IFN space. Let $(Y, \mathcal{E}, \mathcal{F})$ be a non-Archimedean intuitionistic fuzzy Banach space. 
Suppose that $f, g$ and h are functions from $X$ to $Y$ such that $f(0)=g(0)=h(0)=0$, and there is an integer $k$ with $|k|<1$ and satisfies

$$
\begin{aligned}
& \mathcal{E}(f(x+y)-g(x)-h(y), t) \geq \mathcal{E}^{\prime}\left(\|x\|^{r}\|y\|^{s} z_{0}, t\right), \\
& \mathcal{F}(f(x+y)-g(x)-h(y), t) \leq \mathcal{F}^{\prime}\left(\|x\|^{r}\|y\|^{s} z_{\circ}, t\right),
\end{aligned}
$$

for all $x, y \in X, t>0$ and for some fixed $z_{\circ} \in Z$ and $r, s \geq 0$ with $r+s<1$. Then there exists a unique additive mapping $T: X \rightarrow Y$ such that

$$
\begin{aligned}
& \mathcal{E}(f(x)-T(x), t) \geq \mathcal{E}^{\prime}\left((k-1)^{s}\|x\|^{r+s} z_{\circ},|k|^{r+s} t\right), \\
& \mathcal{F}(f(x)-T(x), t) \leq \mathcal{F}^{\prime}\left((k-1)^{s}\|x\|^{r+s} z_{\circ},|k|^{r+s} t\right), \\
& \mathcal{E}(g(x)-T(x), t) \geq \mathcal{E}^{\prime}\left((k-1)^{s}\|x\|^{r+s} z_{\circ},|k|^{r+s} t\right), \\
& \mathcal{F}(g(x)-T(x), t) \leq \mathcal{F}^{\prime}\left((k-1)^{s}\|x\|^{r+s} z_{\circ},|k|^{r+s} t\right), \\
& \mathcal{E}(h(x)-T(x), t) \geq \mathcal{E}^{\prime}\left((k-1)^{s}\|x\|^{r+s} z_{\circ},|k|^{r+s} t\right), \\
& \mathcal{F}(h(x)-T(x), t) \leq \mathcal{F}^{\prime}\left((k-1)^{s}\|x\|^{r+s} z_{\circ},|k|^{r+s} t\right),
\end{aligned}
$$

for all $x \in X$ and $t>0$.

Proof. Let the function $\varphi: X \times X \rightarrow Z$ be defined by $\varphi(x, y)=\|x\|^{r}\|y\|^{s} z_{0}$ for all $x, y \in X$ and $z_{\circ}$ is a fixed unit vector in $Z$. Then (4.1) holds. Since

$$
\begin{gathered}
\mathcal{E}^{\prime}\left(\varphi\left(k^{-1} x, k^{-1} y\right), t\right)=\mathcal{E}^{\prime}\left(\left(\left\|k^{-1} x\right\|^{r}\left\|k^{-1} y\right\|^{s}\right) z_{\circ}, t\right)=\mathcal{E}^{\prime}\left(\left(\|x\|^{r}\|y\|^{s}\right) z_{\circ},|k|^{r+s} t\right), \\
\mathcal{F}^{\prime}\left(\varphi\left(k^{-1} x, k^{-1} y\right), t\right)=\mathcal{F}^{\prime}\left(\left(\|x\|^{r}\|y\|^{s}\right) z_{\circ},|k|^{r+s} t\right),
\end{gathered}
$$

for each $x, y \in X$ and $t>0$. If $\alpha=|k|^{r+s}$ and $r+s<1$, then $\alpha>|k|$ holds. It follows from Theorem 4.1 that there exists a unique additive mapping $C: X \rightarrow Y$ such that (4.3)-(4.5) hold.

\section{Stability of Jensen Functional Equation}

The stability problem for the Jensen functional equation was first proved by Kominek [13] and since then several generalizations and applications of this notion have been investigated by various authors, namely, Jung [12], Mohiuddine [23], Parnami and Vasudeva [41], and many others. The Jensen functional equation is $2 f((x+y) / 2)=f(x)+f(y)$, where $f$ is a mapping between linear spaces. It is easy to see that a mapping $f: X \rightarrow Y$ between linear spaces with $f(0)=0$ satisfies the Jensen equation if and only if it is additive (cf. [41]).

Theorem 5.1. Let $X$ be a linear space over a non-Archimedean field $\mathbb{K}$ and let $\left(Z, \mathcal{\varepsilon}^{\prime}, \mathcal{F}^{\prime}\right)$ be a nonArchimedean IFN space. Suppose that $\varphi: X \times X \rightarrow Z$ is a function such that for some $\alpha>0$ and 
some positive integer $k$ with $|k|<\alpha$ satisfies (3.1). Suppose that $(Y, \mathcal{\varepsilon}, \mathcal{F})$ is a non-Archimedean intuitionistic fuzzy Banach space. If a map $f: X \rightarrow Y$ satisfies

$$
\begin{aligned}
& \mathcal{\varepsilon}\left(2 f\left(\frac{x+y}{2}\right)-f(x)-f(y), t\right) \geq \mathcal{E}^{\prime}(\varphi(x, y), t), \\
& \mathcal{F}\left(2 f\left(\frac{x+y}{2}\right)-f(x)-f(y), t\right) \leq \mathcal{F}^{\prime}(\varphi(x, y), t),
\end{aligned}
$$

for all $x, y \in X$ and $t>0$, then there exists a unique additive mapping $C: X \rightarrow Y$ such that

$$
\mathcal{E}(f(x)-f(0)-C(x), t) \geq \mathcal{M}(x, \alpha t), \quad F(f(x)-f(0)-C(x), t) \leq \mathcal{N}(x, \alpha t),
$$

for all $x \in X$ and $t>0$, where

$$
\begin{aligned}
\mathcal{M}(x, t)= & \mathcal{E}^{\prime}(\varphi(x, x), t) * \mathcal{E}^{\prime}(\varphi(x, 2 x), t) * \cdots * \mathcal{E}^{\prime}(\varphi(x,(k-1) x), t) * \mathcal{E}^{\prime}(\varphi(2 x, 0), t) \\
& * \mathcal{E}^{\prime}(\varphi(3 x, 0), t) * \cdots * \mathcal{E}^{\prime}(\varphi(k x, 0), t), \\
\mathcal{N}(x, t)= & \mathcal{F}^{\prime}(\varphi(x, x), t) \diamond \mathcal{F}^{\prime}(\varphi(x, 2 x), t) \diamond \cdots \diamond \mathcal{F}^{\prime}(\varphi(x,(k-1) x), t) \diamond \mathcal{F}^{\prime}(\varphi(2 x, 0), t) \\
& \diamond \mathcal{F}^{\prime}(\varphi(3 x, 0), t) \diamond \cdots \diamond \mathcal{F}^{\prime}(\varphi(k x, 0), t) .
\end{aligned}
$$

Proof. Suppose that $g(x)=f(x)-f(0)$ for all $x \in X$. Then

$$
\begin{aligned}
& \mathcal{E}\left(2 g\left(\frac{x+y}{2}\right)-g(x)-g(y), t\right) \geq \mathcal{E}^{\prime}(\varphi(x, y), t), \\
& \mathcal{F}\left(2 g\left(\frac{x+y}{2}\right)-g(x)-g(y), t\right) \leq \mathcal{F}^{\prime}(\varphi(x, y), t),
\end{aligned}
$$

for all $x, y \in X$ and $t>0$. Replacing $x$ by $x+y$ and $y$ by 0 in (5.5), then, for all $x, y \in X$ and $t>0$, we have

$$
\begin{aligned}
& \mathcal{E}\left(2 g\left(\frac{x+y}{2}\right)-g(x+y), t\right) \geq \mathcal{E}^{\prime}(\varphi(x+y, 0), t), \\
& \mathcal{F}\left(2 g\left(\frac{x+y}{2}\right)-g(x+y), t\right) \leq \mathcal{F}^{\prime}(\varphi(x+y, 0), t) .
\end{aligned}
$$

From (5.5) and (5.6), we conclude that

$$
\begin{aligned}
& \mathcal{\varepsilon}(g(x+y)-g(x)-g(y), t) \geq \mathcal{E}^{\prime}(\varphi(x, y), t) * \mathcal{E}^{\prime}(\varphi(x+y, 0), t), \\
& \mathcal{F}(g(x+y)-g(x)-g(y), t) \leq \mathcal{F}^{\prime}(\varphi(x, y), t) \diamond F^{\prime}(\varphi(x+y, 0), t),
\end{aligned}
$$


for all $x, y \in X$ and $t>0$. Proceeding the same lines as in the proof of Theorem 3.1, one can show that there exists a unique additive mapping $C: X \rightarrow Y$ such that

$$
\begin{aligned}
& \mathcal{E}(f(x)-f(0)-C(x), t)=\mathcal{E}(g(x)-T(x), \alpha t) \geq \mathcal{M}(x, t), \\
& \mathcal{F}(f(x)-f(0)-C(x), t)=\mathcal{F}(g(x)-T(x), \alpha t) \leq \mathcal{N}(x, t),
\end{aligned}
$$

for all $x \in X$ and $t>0$.

\section{Stability of Pexiderized Jensen Functional Equation}

The functional equation $2 f((x+y) / 2)=g(x)+h(y)$ is said to be Pexiderized Jensen, where $f, g$, and $h$ are mappings between linear spaces. In the case $f=g=h$, it is called Jensen functional equation.

Theorem 6.1. Let $X$ be a linear space over a non-Archimedean field $\mathbb{K}$ and let $(Y, \mathcal{\varepsilon}, \boldsymbol{F})$ be a nonArchimedean intuitionistic fuzzy Banach space. Suppose that $f, g$, and $h$ are mappings from $X$ to $Y$ with $f(0)=g(0)=h(0)=0$. Let $\left(Z, \boldsymbol{E}^{\prime}, \boldsymbol{F}^{\prime}\right)$ be non-Archimedean IFN space. Suppose that $\varphi$ : $X \times X \rightarrow Z$ is a function such that for some $\alpha>0$, and some positive integer $k$ with $|k|<\alpha$ satisfies (3.1) and inequality

$$
\begin{aligned}
& \mathcal{E}\left(2 f\left(\frac{x+y}{2}\right)-g(x)-h(y), t\right) \geq \mathcal{E}^{\prime}(\varphi(x, y), t), \\
& \mathcal{F}\left(2 f\left(\frac{x+y}{2}\right)-g(x)-h(y), t\right) \leq \mathcal{F}^{\prime}(\varphi(x, y), t),
\end{aligned}
$$

for all $x, y \in X$ and $t>0$. Then there exists a unique additive mapping $C: X \rightarrow Y$ such that

$$
\begin{gathered}
\mathcal{E}(f(x)-C(x), t) \geq \mathcal{M}(x, \alpha t), \quad \mathcal{F}(f(x)-C(x), t) \leq \mathcal{N}(x, \alpha t), \\
\mathcal{E}(g(x)-C(x), t) \geq \mathcal{M}\left(\frac{x}{2}, \frac{\alpha t}{2}\right) * \mathcal{E}^{\prime}(\varphi(x, 0), t), \\
\mathcal{F}(g(x)-C(x), t) \leq \mathcal{N}\left(\frac{x}{2}, \frac{\alpha t}{2}\right) \diamond \mathcal{F}^{\prime}(\varphi(x, 0), t), \\
\mathcal{E}(h(x)-C(x), t) \geq \mathcal{M}\left(\frac{x}{2}, \frac{\alpha t}{2}\right) * \mathcal{E}^{\prime}(\varphi(0, x), t), \\
\mathcal{F}(h(x)-C(x), t) \leq \mathcal{N}\left(\frac{x}{2}, \frac{\alpha t}{2}\right) \diamond \mathcal{F}^{\prime}(\varphi(0, x), t),
\end{gathered}
$$


for all $x \in X$ and $t>0$, where

$$
\begin{array}{r}
\mathcal{M}(x, t)=\prod_{m=1}^{k-1}\left\{\mathcal{E}^{\prime}(\varphi(x, m x),|2| t) * \mathcal{E}^{\prime}(\varphi(m x, m x),|2| t)\right\} \\
* \prod_{m=0}^{k}\left\{\mathcal{E}^{\prime}(\varphi(m x, 0),|2| t) * \mathcal{E}^{\prime}(\varphi(0, m x),|2| t)\right\}, \\
\mathcal{N}(x, t)=\coprod_{m=1}^{k-1}\left\{\mathcal{E}^{\prime}(\varphi(x, m x),|2| t) \diamond \mathcal{E}^{\prime}(\varphi(m x, m x),|2| t)\right\} \\
\diamond \coprod_{m=0}^{k}\left\{\mathcal{E}^{\prime}(\varphi(m x, 0),|2| t) \diamond \mathcal{E}^{\prime}(\varphi(0, m x),|2| t)\right\} .
\end{array}
$$

Proof. Put $y=x$ in (6.1). Then, for all $x \in X$ and $t>0$

$$
\begin{aligned}
& \mathcal{E}(2 f(x)-g(x)-h(x), t) \geq \mathcal{E}^{\prime}(\varphi(x, x), t), \\
& \mathcal{F}(2 f(x)-g(x)-h(x), t) \leq \mathcal{F}^{\prime}(\varphi(x, x), t) .
\end{aligned}
$$

Replacing $x$ by $y$ in (6.1), we get

$$
\begin{aligned}
& \mathcal{\varepsilon}(2 f(y)-g(y)-h(y), t) \geq \mathcal{E}^{\prime}(\varphi(y, y), t), \\
& \mathcal{F}(2 f(y)-g(y)-h(y), t) \leq \mathcal{F}^{\prime}(\varphi(y, y), t),
\end{aligned}
$$

for all $y \in X$ and $t>0$. Again replacing $x$ by $y$ as well as $y$ by $x$ in (6.1), we get

$$
\begin{aligned}
& \mathcal{E}\left(2 f\left(\frac{x+y}{2}\right)-g(y)-h(x), t\right) \geq \mathcal{E}^{\prime}(\varphi(y, x), t), \\
& \mathcal{F}\left(2 f\left(\frac{x+y}{2}\right)-g(y)-h(x), t\right) \leq \mathcal{F}^{\prime}(\varphi(y, x), t),
\end{aligned}
$$

for all $x, y \in X$ and $t>0$. It follows from (6.1) and (6.6)-(6.8) that

$$
\begin{aligned}
& \mathcal{\varepsilon}\left(4 f\left(\frac{x+y}{2}\right)-2 f(x)-2 f(y), t\right) \\
& \quad \geq \mathcal{E}^{\prime}(\varphi(x, x), t) * \mathcal{E}^{\prime}(\varphi(x, y), t) * \mathcal{E}^{\prime}(\varphi(y, y), t) * \mathcal{E}^{\prime}(\varphi(y, x), t), \\
& \mathcal{F}\left(4 f\left(\frac{x+y}{2}\right)-2 f(x)-2 f(y), t\right) \\
& \quad \leq \mathcal{F}^{\prime}(\varphi(x, x), t) \diamond \mathcal{F}^{\prime}(\varphi(x, y), t) \diamond \mathcal{F}^{\prime}(\varphi(y, y), t) \diamond \mathcal{F}^{\prime}(\varphi(y, x), t) .
\end{aligned}
$$


Thus, for all $x, y \in X$ and $t>0$,

$$
\begin{aligned}
& \mathcal{E}\left(2 f\left(\frac{x+y}{2}\right)-f(x)-f(y), t\right) \\
& \quad \geq \mathcal{E}^{\prime}(\varphi(x, x),|2| t) * \mathcal{E}^{\prime}(\varphi(x, y),|2| t) * \mathcal{E}^{\prime}(\varphi(y, y),|2| t) * \mathcal{E}^{\prime}(\varphi(y, x),|2| t), \\
& \mathcal{F}\left(2 f\left(\frac{x+y}{2}\right)-f(x)-f(y), t\right) \\
& \quad \leq \mathcal{F}^{\prime}(\varphi(x, x),|2| t) \diamond \mathcal{F}^{\prime}(\varphi(x, y),|2| t) \diamond \mathcal{F}^{\prime}(\varphi(y, y),|2| t) \diamond \mathcal{F}^{\prime}(\varphi(y, x),|2| t) .
\end{aligned}
$$

Proceeding the same argument used in Theorem 5.1 shows that there exists a unique additive mapping $C: X \rightarrow Y$ such that (6.2) holds. Therefore

$$
\mathcal{E}\left(2 f\left(\frac{x}{2}\right)-C(x), t\right) \geq \mathcal{M}\left(\frac{x}{2}, \frac{\alpha t}{2}\right), \quad \mp\left(2 f\left(\frac{x}{2}\right)-C(x), t\right) \leq \mathcal{N}\left(\frac{x}{2}, \frac{\alpha t}{2}\right),
$$

for all $x \in X$ and $t>0$. Put $y=0$ in (6.1), we get

$$
\varepsilon\left(2 f\left(\frac{x}{2}\right)-g(x), t\right) \geq \mathcal{E}^{\prime}(\varphi(x, 0), t), \quad \mp\left(2 f\left(\frac{x}{2}\right)-g(x), t\right) \leq \mathcal{F}^{\prime}(\varphi(x, 0), t),
$$

for all $x \in X$ and $t>0$. It follows from (6.11) and (6.12) that (6.3) holds. Similarly we can show that (6.4) holds.

Corollary 6.2. Let $X$ be a non-Archimedean normed space. Suppose that $f, g, h: X \rightarrow Y$ such that $f(0)=g(0)=h(0)=0$, and there is an integer $k$ with $|k|<1$ and satisfies

$$
\left\|2 f\left(\frac{x+y}{2}\right)-g(x)-h(y)\right\| \leq \epsilon,
$$

for all $x, y \in X$. Then there exists a unique additive mapping $C: X \rightarrow Y$ such that

$$
\|f(x)-C(x)\| \leq \epsilon, \quad\|g(x)-C(x)\| \leq \epsilon, \quad\|h(x)-C(x)\| \leq \epsilon,
$$

for all $x \in X$.

Proof. Let the function $\mathcal{E}, \mathcal{F}: \mathbb{Y} \times \mathbb{R} \rightarrow[0,1]$ be defined by

$$
\mathcal{E}(x, t)=\left\{\begin{array}{ll}
\frac{t}{t+\|x\|} & \text { if } t>0, \\
0 & \text { if } t \leq 0 ;
\end{array} \quad \mp(x, t)= \begin{cases}\frac{\|x\|}{t+\|x\|} & \text { if } t>0, \\
1 & \text { if } t \leq 0,\end{cases}\right.
$$


on $Y$. It is easy to see that $(Y, \mathcal{E}, \mathcal{F})$ is a non-Archimedean intuitionistic fuzzy Banach space. Consider the non-Archimedean intuitionistic fuzzy norm

$$
\mathcal{E}^{\prime}(z, t)=\left\{\begin{array}{ll}
\frac{t}{t+|z|} & \text { if } t>0, \\
0 & \text { if } t \leq 0 ;
\end{array} \quad \mathbb{F}^{\prime}(z, t)= \begin{cases}\frac{|z|}{t+|z|} & \text { if } t>0 \\
1 & \text { if } t \leq 0\end{cases}\right.
$$

Then $\left(\mathcal{E}^{\prime}, \mathcal{F}^{\prime}\right)$ is a non-Archimedean intuitionistic fuzzy norm on $\mathbb{R}$. It is easy to see that (4.1) holds for $\varphi(x, y)=\epsilon$ and $\alpha=1$ satisfies (3.1). Therefore the condition of Theorem 6.1 is fulfilled. Hence there exists a unique additive mapping $C: X \rightarrow Y$ such that (6.14) holds.

\section{References}

[1] S. M. Ulam, A Collection of Mathematical Problems, vol. 8 of Interscience Tracts in Pure and Applied Mathematics, Interscience Publishers, New York, NY, USA, 1960.

[2] D. H. Hyers, "On the stability of the linear functional equation," Proceedings of the National Academy of Sciences of the United States of America, vol. 27, pp. 222-224, 1941.

[3] T. Aoki, "On the stability of the linear transformation in Banach spaces," Journal of the Mathematical Society of Japan, vol. 2, pp. 64-66, 1950.

[4] T. M. Rassias, "On the stability of the linear mapping in Banach spaces," Proceedings of the American Mathematical Society, vol. 72, no. 2, pp. 297-300, 1978.

[5] Z. Gajda, "On stability of additive mappings," International Journal of Mathematics and Mathematical Sciences, vol. 14, no. 3, pp. 431-434, 1991.

[6] P. Găvruţa, "A generalization of the Hyers-Ulam-Rassias stability of approximately additive mappings," Journal of Mathematical Analysis and Applications, vol. 184, no. 3, pp. 431-436, 1994.

[7] M. E. Gordji, H. Khodaei, and R. Khodabakhsh, "General quartic-cubic-quadratic functional equation in non-Archimedean normed spaces," Politehnica University of Bucharest Scientific Bulletin A, vol. 72, no. 3, pp. 69-84, 2010.

[8] M. E. Gordji, H. Khodaei, and M. Kamyar, "Stability of Cauchy-Jensen type functional equation in generalized fuzzy normed spaces," Computers \& Mathematics with Applications, vol. 62, no. 8, pp. 29502960, 2011.

[9] M. Eshaghi Gordji and M. B. Savadkouhi, "Stability of cubic and quartic functional equations in nonArchimedean spaces," Acta Applicandae Mathematicae, vol. 110, no. 3, pp. 1321-1329, 2010.

[10] M. Eshaghi Gordji and M. B. Savadkouhi, "Stability of a mixed type cubic-quartic functional equation in non-Archimedean spaces," Applied Mathematics Letters, vol. 23, no. 10, pp. 1198-1202, 2010.

[11] D. H. Hyers, G. Isac, and T. M. Rassias, Stability of Functional Equations in Several Variables, vol. 34 of Progress in Nonlinear Differential Equations and Their Applications, Birkhäuser, Boston, Mass, USA, 1998.

[12] S.-M. Jung, "Hyers-Ulam-Rassias stability of Jensen's equation and its application," Proceedings of the American Mathematical Society, vol. 126, no. 11, pp. 3137-3143, 1998.

[13] Z. Kominek, “On a local stability of the Jensen functional equation," Demonstratio Mathematica, vol. 22, no. 2, pp. 499-507, 1989.

[14] Y.-H. Lee and K.-W. Jun, "A generalization of the Hyers-Ulam-Rassias stability of Jensen's equation," Journal of Mathematical Analysis and Applications, vol. 238, no. 1, pp. 305-315, 1999.

[15] A. K. Mirmostafaee and M. S. Moslehian, "Stability of additive mappings in non-Archimedean fuzzy normed spaces," Fuzzy Sets and Systems, vol. 160, no. 11, pp. 1643-1652, 2009.

[16] T. M. Rassias, "On the stability of functional equations and a problem of Ulam," Acta Applicandae Mathematicae, vol. 62, no. 1, pp. 23-130, 2000.

[17] C. Park, "Fixed points and Hyers-Ulam-Rassias stability of Cauchy-Jensen functional equations in Banach algebras," Fixed Point Theory and Applications, vol. 2007, Article ID 50175, 15 pages, 2007.

[18] T. Z. Xu, J. M. Rassias, and W. X. Xu, "Intuitionistic fuzzy stability of a general mixed additive-cubic equation," Journal of Mathematical Physics, vol. 51, no. 6, Article ID 063519, 2010.

[19] T. Z. Xu, J. M. Rassias, and W. X. Xu, "Stability of a general mixed additive-cubic functional equation in non-Archimedean fuzzy normed spaces," Journal of Mathematical Physics, vol. 51, no. 9, Article ID 093508, 2010. 
[20] S. A. Mohiuddine and A. Alotaibi, "Fuzzy stability of a cubic functional equation via fixed point technique," Advances in Difference Equations, vol. 2012, article 48, 2012.

[21] S. A. Mohiuddine and H. Ševli, "Stability of Pexiderized quadratic functional equation in intuitionistic fuzzy normed space," Journal of Computational and Applied Mathematics, vol. 235, no. 8, pp. 21372146, 2011.

[22] S. A. Mohiuddine, M. Cancan, and H. Şevli, "Intuitionistic fuzzy stability of a Jensen functional equation via fixed point technique," Mathematical and Computer Modelling, vol. 54, no. 9-10, pp. 2403-2409, 2011.

[23] S. A. Mohiuddine, "Stability of Jensen functional equation in intuitionistic fuzzy normed space," Chaos, Solitons \& Fractals, vol. 42, no. 5, pp. 2989-2996, 2009.

[24] M. Mursaleen and S. A. Mohiuddine, "On stability of a cubic functional equation in intuitionistic fuzzy normed spaces," Chaos, Solitons E Fractals, vol. 42, no. 5, pp. 2997-3005, 2009.

[25] Z. Wang and T. M. Rassias, "Intuitionistic fuzzy stability of functional equations associated with inner product spaces," Abstract and Applied Analysis, vol. 2011, Article ID 456182, 19 pages, 2011.

[26] S. A. Mohiuddine and M. A. Alghamdi, "Stability of functional equation obtained through fixed point alternative in intuitionistic fuzzy normed spaces," Advances in Difference Equations, vol. 2012, article 141, 2012.

[27] R. Saadati and J. H. Park, "On the intuitionistic fuzzy topological spaces," Chaos, Solitons and Fractals, vol. 27, no. 2, pp. 331-344, 2006.

[28] S. A. Mohiuddine and Q. M. D. Lohani, "On generalized statistical convergence in intuitionistic fuzzy normed space," Chaos, Solitons \& Fractals, vol. 42, no. 3, pp. 1731-1737, 2009.

[29] M. Mursaleen and S. A. Mohiuddine, "Statistical convergence of double sequences in intuitionistic fuzzy normed spaces," Chaos, Solitons \& Fractals, vol. 41, no. 5, pp. 2414-2421, 2009.

[30] M. Mursaleen and S. A. Mohiuddine, "On lacunary statistical convergence with respect to the intuitionistic fuzzy normed space," Journal of Computational and Applied Mathematics, vol. 233, no. 2, pp. 142-149, 2009.

[31] M. Mursaleen and S. A. Mohiuddine, "Nonlinear operators between intuitionistic fuzzy normed spaces and Fréchet derivative," Chaos, Solitons \& Fractals, vol. 42, no. 2, pp. 1010-1015, 2009.

[32] M. Mursaleen, S. A. Mohiuddine, and O. H. H. Edely, “On the ideal convergence of double sequences in intuitionistic fuzzy normed spaces," Computers \& Mathematics with Applications, vol. 59, no. 2, pp. 603-611, 2010.

[33] Y. Y1lmaz, "On some basic properties of differentiation in intuitionistic fuzzy normed spaces," Mathematical and Computer Modelling, vol. 52, no. 3-4, pp. 448-458, 2010.

[34] M. Mursaleen, V. Karakaya, and S. A. Mohiuddine, "Schauder basis, separability, and approximation property in intuitionistic fuzzy normed space," Abstract and Applied Analysis, vol. 2010, Article ID 131868, 14 pages, 2010.

[35] A. Alotaibi and S. A. Mohiuddine, "On the stability of a cubic functional equation in random 2normed spaces," Advances in Difference Equations, vol. 2012, article 39, 2012.

[36] I. Goleț, "On probabilistic 2-normed spaces," Novi Sad Journal of Mathematics, vol. 35, no. 1, pp. 95-102, 2005.

[37] S. A. Mohiuddine and M. Aiyub, "Lacunary statistical convergence in random 2-normed spaces," Applied Mathematics \& Information Sciences, vol. 6, no. 3, pp. 581-585, 2012.

[38] M. Mursaleen, "On statistical convergence in random 2-normed spaces," Acta Universitatis Szegediensis, vol. 76, no. 1-2, pp. 101-109, 2010.

[39] S. A. Mohiuddine, A. Alotaibi, and S. M. Alsulami, "Ideal convergence of double sequences in random 2-normed spaces," Advances in Difference Equations, vol. 2012, article 149, 2012.

[40] K. Hensel, “Uber eine neue Begrndung der Theorie der algebraischen Zahlen, Jahresber," Deutsche Mathematiker-Vereinigung, vol. 6, pp. 83-88, 1897.

[41] J. C. Parnami and H. L. Vasudeva, “On Jensen's functional equation," Aequationes Mathematicae, vol. 43, no. 2-3, pp. 211-218, 1992. 


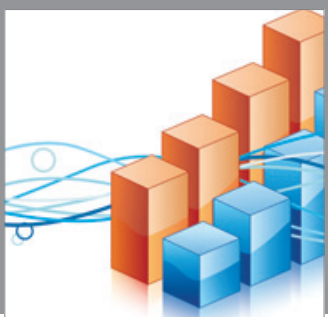

Advances in

Operations Research

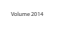

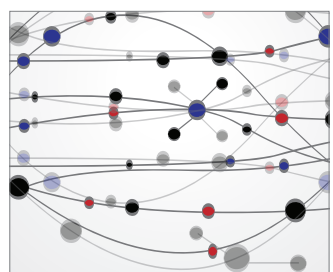

\section{The Scientific} World Journal
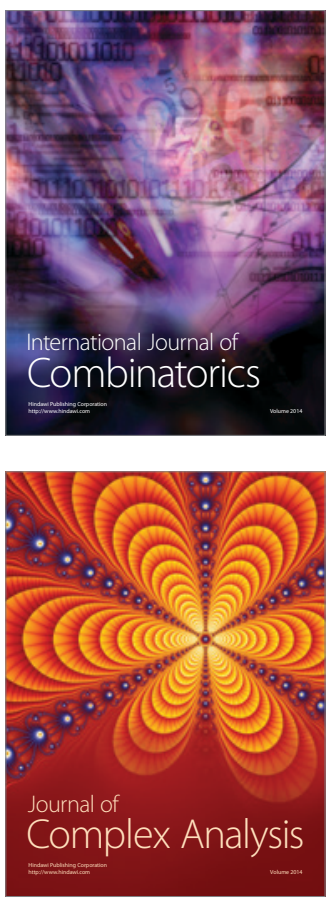

International Journal of

Mathematics and

Mathematical

Sciences
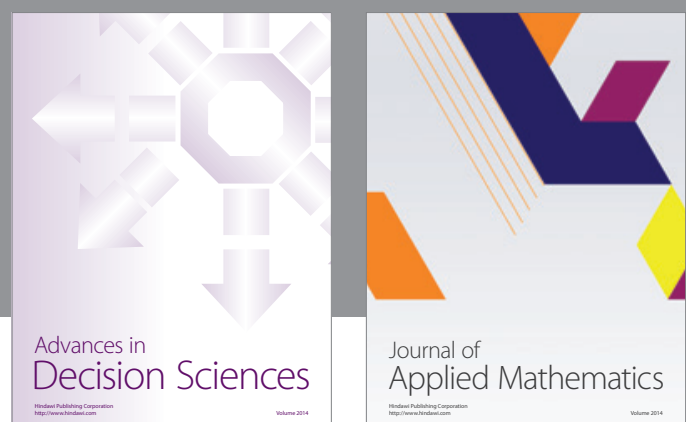

Journal of

Applied Mathematics
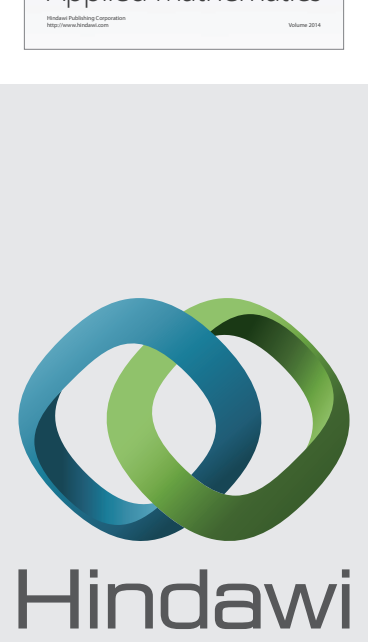

Submit your manuscripts at http://www.hindawi.com
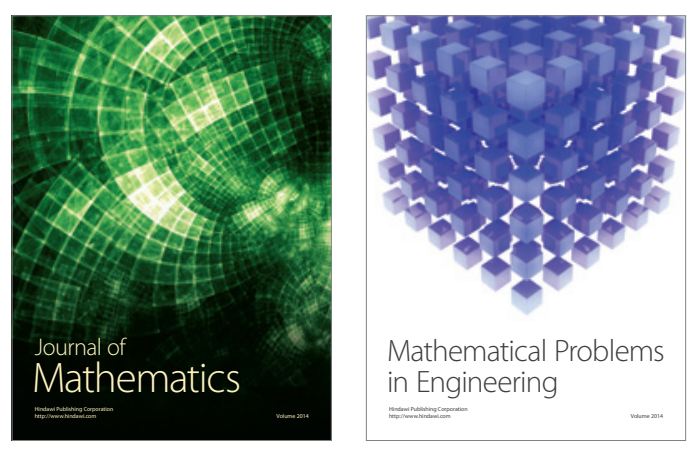

Mathematical Problems in Engineering
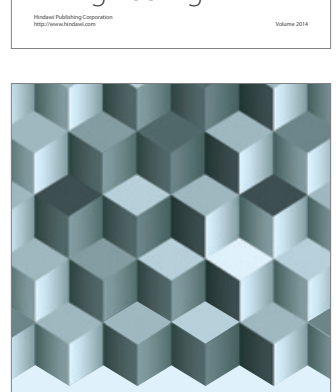

Journal of

Function Spaces
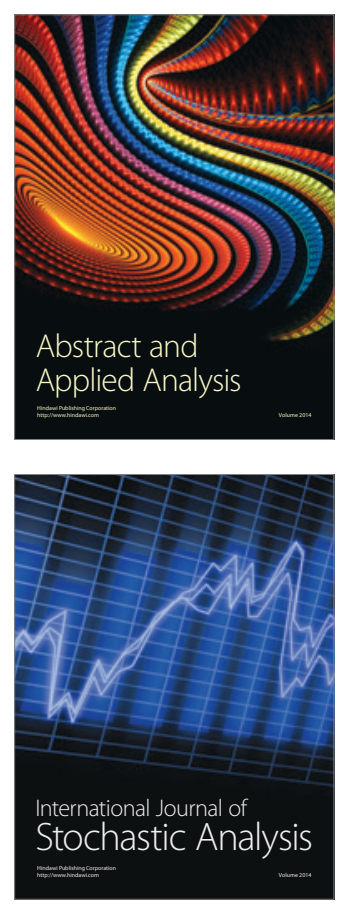

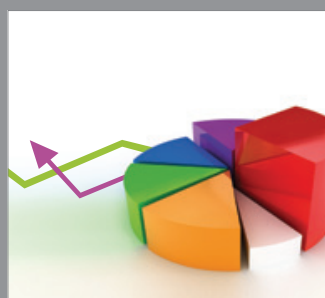

ournal of

Probability and Statistics

Promensencen
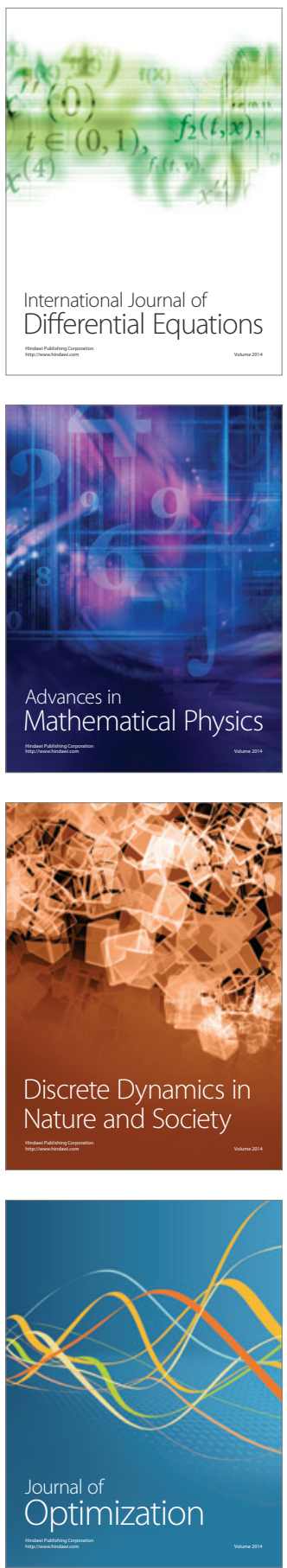